\title{
Typ-2-Diabetes \\ Blutzuckersenkung nicht um jeden Preis
}

— Hypoglykämierisiko, Gewichtserhöhung sowie Verträglichkeitsprobleme sind die Stolpersteine auf dem oft mühsamen Weg zur medikamentösen Blutzuckerkontrolle beim Typ-2-Diabetiker. Nach den ernüchternden Ergebnissen großer Interventionsstudien wie ACCORD, ADVANCE und VADT ist das Ziel der maximalen $\mathrm{HbA}_{1 \mathrm{c}}$-Absenkung „out“. Werte von $6,5 \%$ gegenüber $7,0 \%$ sollen einer DDGStellungnahme zufolge nur noch dann angestrebt werden, wenn Hypoglykämien weitgehend vermieden werden und keine wesentliche Gewichtszunahme auftritt. Wenig untersuchte Mehrfachkombinationen oraler Antidiabetika (in der Regel mehr als zwei) sind nicht zu empfehlen, insbesondere nicht bei zusätzlicher Insulingabe. „Man kann die Kombination auch übertreiben“, warnte Prof. Hans-Georg Joost, Potsdam.

\section{Weniger Neben- und \\ Wechselwirkungen}

Basismedikament für den Typ-2-Diabetes bleibt Metformin. Wird es mit einem Sulfonylharnstoff kombiniert, drohen Hypoglykämien und Gewichtszunahme. Wesentlich geringer ist das Risiko für beides laut Joost bei der Kombination mit einem DPP-4-Hemmer wie Vildagliptin (Galvus ${ }^{\circledR}$ oder als Fixkombination Eucreas ${ }^{\circledR}$ ). Vilda- gliptin ist zudem der einzige zugelassene DPP-4-Hemmer, bei dem keine Interaktionen mit anderen Medikamenten über CYP450 zu befürchten sind.

Antihyperglykämische Wirksamkeit bewiesen hat Vildagliptin auch bei Sekundärversagen von Sulfonylharnstoffen sowie bei unzureichender Insulineinstellung. Gleichzeitig senkt die Zugabe deutlich die Hypoglykämiegefahr. Im Vergleich zur Metforminmonotherapie zeichnet sich der DPP-4-Hemmer laut Prof. Rüdiger Göke, Kirchhain, durch eine bessere gastrointestinale Verträglichkeit aus. Sein Einsatz ist auch bei moderater Niereninsuffizienz möglich.

\section{Nachgefragt}

\section{Johanniskraut und Sonne - verträgt sich das?}

\begin{abstract}
Frage: Immer wieder ist zu hören oder zu lesen, dass Johanniskraut-Präparate photosensibilisierend wirken und demzufolge im Sommer abgesetzt werden sollten. Behauptung oder Tatsache?
\end{abstract}

Prof. Dingermann: Behauptung. Die in der antidepressiven Behandlung eingesetzten Tagesdosen von Johanniskraut - Standard sind $900 \mathrm{mg}$ - sind in aller Regel zu gering, um zu einer signifikant erhöhten Photosensitivität der Haut gegenüber UV-Licht führen zu können.

Prof. Schauder: Tatsache ist, dass Johanniskraut als Auslöser phototoxischer Effekte beim Menschen erheblich überschätzt wird: Bei Gabe als Antidepressivum wurde bisher erst in einem einzigen Fall über eine erhöhte dermale Lichtempfindlichkeit in Form von Rötung und Juckreiz unter Sonneneinwirkung berichtet - und das ist 13 Jahre her.

Frage: Und das generelle Vorkommen medikamenteninduzierter Photosensitivität? Wie viele Arzneistoffe sind als Photosensibilisatoren auf dem deutschen Markt gelistet? Prof. Dingermann: Derzeit sind bei uns ungefähr zoo Arzneistoffe als Auslöser phototoxischer Reaktionen bekannt - darunter auch zahlreiche synthetische Antidepressiva. Wenn man die alle im Sommer absetzen würde ...

Prof. Schauder: Die Prävalenz medikamenteninduzierter Photosensibilisierungen hängt ja nicht nur von der phototoxischen Potenz eines Arzneimittels ab, sondern auch von dessen Verordnungshäufigkeit. Von Johanniskraut werden jährlich etwa 104 Millionen Tagesdosen in der antidepressiven
Therapie eingesetzt, und dennoch gibt es nur diesen einen gemeldeten Fall vor 13 Jahren!

Frage: Kennen Sie aus Ihrer täglichen Praxis Patienten mit sonnenbrandähnlichen Erscheinungen nach Johanniskrautgabe?

Prof. Müller: Keinen Einzigen. Aus der Literatur ist

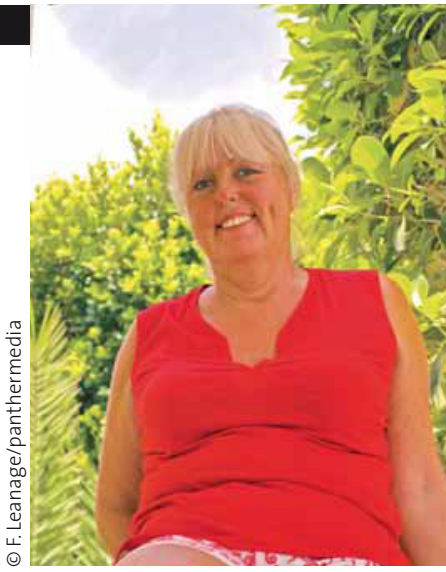
Eine Johanniskrauttherapie ist auch im Sommer sicher. es mir auch nur bei hellhäutigen Weidetieren bekannt. Aber bei meinen Patienten habe ich das in meiner langjährigen Praxis nicht erlebt.

Prof. Volz: Im klinischen Bereich ist mir so etwas auch noch nicht untergekommen. Ich verlasse mich da im Übrigen völlig auf die aktuelle Datenlage, wonach Hypericumextrakt nur bei extremer Überdosierung - jenseits der antidepressiven Therapie-eine Photosensitivität auslösen könnte.

- Interview: Marianne E. Tippmann

Expertenrunde:

Prof. Dr. Theodor Dingermann, Pharmazeutische Biologie, Johann Wolfgang Goethe-Universität Frankfurt am Main

Prof. Dr. Diethard Müller, Niedergelassener Arzt, Ilmenau

Prof. Dr. Silvia Schauder, FA Dermatologie und Venerologie, Göttingen Prof. Dr. Hans-Peter Volz, Ärztl. Direktor des Krankenhauses für Psychiatrie, Psychotherapie und Psychosomatische Medizin, Schloss Werneck 


\section{"Traumpille" Bewegung}

Mindestens ebenso wichtig wie eine optimale Pharmakotherapie ist für den Typ-2Diabetiker eine Lebensstiländerung, um Gewicht und Hypoglykämierisiko in den Griff zu bekommen. „Bewegung ist die Traumpille“, sagte Prof. Peter Schwarz, Dresden. Hinzukommen muss eine Gewichtsreduktion um 5-7\% sowie eine ballaststoffreiche, fettreduzierte Ernährung.

Mit der von der Firma Novartis unterstützten Initiative „Gemeinsam geht es leichter" soll die Kommunikation zwi- schen Arzt und Patient und damit die Motivation der Patienten zu einer nachhaltigen Umstellung ihres Lebensstils verbessert werden. Das „Aktivbuch Diabetes", das als CD-ROM und als Broschüre mit begleitendem Filmmaterial kostenlos zur Verfügung steht, gibt wertvolle Ratschläge. Ausführliche Informationen: www.gemeinsam-geht-es-leichter.de.

- Ursula Einecke

Quelle: Symposium und Pressegespräch,

DDG-Jahrestagung, Stuttgart, 12. Mai 2010

(Veranstalter: Novartis Pharma).

\section{Neuartiges Antianginosum ist Kandidat für Galenus-Preis Optimierter Ischämieschutz für KHK-Patienten}

— Im Oktober verleiht die Ärzte Zeitung den Galenus von Pergamon-Preis 2010 für eine herausragende Arzneimittelinnovation. Zu den Bewerbern gehört auch das vom Unternehmen Berlin-Chemie eingeführte Ranolazin (Ranexa ${ }^{\circledR}$ ).

Ranolazin ist indiziert zur symptomatischen Therapie bei chronischer stabiler Angina pectoris, wenn antianginöse Basistherapeutika kontraindizert sind bzw. nicht vertragen werden oder als Ergänzung, wenn die Patienten weiter Beschwerden haben. Dabei wirkt Ranolazin direkt in der Herzmuskelzelle, indem es den unter ischämischen Bedingungen erhöhten späten Natriumeinstrom selektiv hemmt. Auf diese Weise werden Angina-pectorisAttacken und Nitratverbrauch gesenkt, ohne dass es zu Veränderungen von Herzfrequenz oder Blutdruck kommt. Damit ist es das erste Antianginosum, das seine Wirkung nicht über eine Veränderung hämodynamischer Parameter entfaltet.

\section{Zusatztherapie reduziert}

\section{Anginaattacken und Nitratverbrauch}

Positive klinische Effekte von Ranolazin bei KHK-Patienten mit wiederkehrenden Ischämien sind in mehreren klinischen Studien bestätigt worden. So verbesserte die Zusatztherapie mit Ranolazin in der Phase-III-Studie CARISA die Leistungsfä- higkeit der Patienten nach zwölf Wochen signifikant. Die Zahl pektanginöser Attacken sank ebenso wie der Verbrauch von kurz wirksamen Nitraten.

Die große, prospektive Interventionsstudie MERLIN-TIMI36 konnte ebenfalls die gute Wirksamkeit von Ranolazin in der Therapie der wiederkehrenden Angina pectoris nachweisen. Die Behandlung additiv zu ASS, Statin oder Betablocker (im Schnitt 2,9 Antianginosa) über einen Zeitraum von durchschnittlich 350 Tagen zeigte in der Subgruppe von Patienten mit ischämischen Attacken eine signifikante antiischämische Wirkung: Gegenüber Placebo wurde die Inzidenz rezidivierender Ischämien um $22 \%$ reduziert. Gleichzeitig sank das Risiko für eine Verschlechterung der Angina pectoris um 31\%.

Bei schweren Myokardischämien mit EKG-Veränderungen oder nachfolgender Klinikeinweisung war eine signifikante Abnahme der Häufigkeit um 19\% zu verzeichnen. Auch die Belastungskapazität wurde im Ranolazinarm signifikant verbessert. Gleichzeitig erwies sich Ranolazin als gut verträglich. Gesamtsterblichkeit und plötzlicher Herztod waren in beiden Gruppe vergleichbar häufig. Klinisch signifikante Arrhythmien im Langzeit-EKG waren signifikant seltener unter Ranolazin. - $\mathrm{OB}$

\section{Online fortbilden und CME-Punkte sammeln}

\author{
Folgende Fortbildungen der $\mathbf{M M} \mathbf{W}$ \\ stehen Ihnen derzeit auf \\ www.CME-Punkt.de zur Verfügung \\ (Auswahl):
}

- Diagnostik und Akuttherapie chronisch entzündlicher Darmerkrankungen

- Der pädiatrische Notfall in der Praxis

- Diagnostik und Therapie der Neuroborreliose

- Impfcheck

- Neu diagnostizierter Typ-2-Diabetes

- Rückenschmerzen

Die Teilnahme ist für Sie völlig kostenfrei. Melden Sie sich heute noch an.

\section{UND SO GEHT'S:}

\ Auf www.CME-Punkt.de gehen und sich einmal registrieren.

\ Dazu geben Sie persönliche Daten und Ihre Einheitliche Fortbildungsnummer (EFN) ein.

$\nabla$ Nach der Registrierung stehen Ihnen sämtliche CME-Fortbildungen der Zeitschriften von Urban\&Vogel kostenfrei zur Verfügung. Für Hausärzte und Internisten besonders interessant: MMW, Der Hausarzt, Medizinische Klinik, GastroNews, Cardiovasc, InFo Diabetologie, PneumoNews.

\ Wenn Sie eine Fortbildungseinheit bestehen (bis zu 3 Punkte), können Sie sich Ihre Teilnahmebestätigung direkt als PDF herunterladen.

V Wir empfehlen, diese Bescheinigungen gesammelt bei lhrer Landesärztekammer einzureichen. 